\title{
The application of stratified medicine to dementia care
}

\author{
Emad Sidhom $\mathbb{D}^{\circ}$, John 0'Brien \& Benjamin R. Underwood
}

\begin{abstract}
SUMMARY
Stratified medicine has been successfully used in many areas of medicine, perhaps most notably oncology. There is now both a growing evidence base and mounting enthusiasm, supported at a governmental level and across industry, academia and clinical medicine, to apply this approach to neurodegenerative illnesses, including dementia, as these provide the greatest clinical and social challenge of our times. In this article we consider definitions of stratified medicine, look at its application in other medical specialties, review the national context in the UK and consider the current state, future potential and specific considerations of applying stratified medicine to dementia.
\end{abstract}

\section{LEARNING OBJECTIVES}

After reading this article you will be able to:

- describe the concept of stratified medicine and its applications in medicine

- consider the potential application of stratified medicine in dementia care

- describe the availability of tests that may be relevant to stratified medicine in dementia care.

\section{KEYWORDS}

Dementia; stratified medicine; neurodegenerative disorders; personalised medicine; genetics.

There is no single uniformly accepted definition of stratified medicine, alternatively called personalised or precision medicine. Instead, a number of different definitions exist. Perhaps the most simple is that adopted by the UK's Academy of Medical Sciences, which defines stratified medicine as 'the grouping of patients based on risk of disease or response to therapy by using diagnostic tests or techniques' (Academy of Medical Sciences 2020). What the different definitions have in common is the concept of grouping patients, often using molecular or genetic techniques, with a view to enhancing their care. That care may include more accurate diagnosis, but more frequently focuses on enhanced safety or effectiveness of treatment. The enthusiasm for this approach comes partly from a desire to maximise the clinical benefit of advances in basic science, for example the Human Genome Project and the advance of 'omic' technologies, but also from the clear benefits this approach has brought in other areas of medicine, notably oncology (Venter 2001; Michels 2016) (Box 1).

Figure 1 highlights the first description of Alzheimer's disease, by Alois Alzheimer in 1906 (Berrios 1990; Stelzmann 1995), the first successful application of stratified medicine in oncology with tamoxifen in 1967 (Jordan 2006), the first positive trial of tacrine in Alzheimer's disease (Summers 1981), the beginning of the Human Genome Project (Moraes 2016), the discovery of mutations in amyloid precursor protein that lead to dementia (Goate 1991), the identification of apolipoprotein $\mathrm{E}(\mathrm{ApoE})$ as a genetic risk factor for Alzheimer's (Corder 1993; Strittmatter 1993), the first study looking at cerebrospinal fluid (CSF) biomarkers (Motter 1995; Blennow 2015) and the discovery of imaging brain amyloid in Alzheimer's disease using Pittsburgh compound-B for positron emission tomography (PET) (Klunk 2004).

It seems intuitive that there will be individual variation in response to treatment. Outside paediatric medicine even doses of drugs are seldom adjusted for body weight or age, although few clinicians would see giving a particular dose of a drug to a $40 \mathrm{~kg}$ 90-year-old woman as equivalent to giving the same dose to a $120 \mathrm{~kg}$ 20-year-old man. Medicine has moved on from simple diagnosis being made on symptom description to encompass markers (such as imaging and genetics) that are more closely related to pathology. In this context the process of making a diagnosis can itself be seen as a form of stratified medicine. This is an important concept for people working clinically: stratified medicine is seen not just as something esoteric for the future, but as something that underpins current clinical practice. The impetus now is to see how this can be built on to improve clinical outcomes, for example by means of further diagnostic subclassification. Lung cancer has traditionally been divided on the basis of histopathology but also increasingly by specific genetic mutations, which can then be used to guide therapy more precisely, improving outcomes for those treated and sparing others exposure to treatments that are not effective (Lenz 2013).
Emad Sidhom is a clinical research associate in the Department of Clinical Neurosciences, University of Cambridge, UK. He is working on modification of the unfolded protein response in neurodegenerative disorders. He is interested in research on low-amplitude, ultra-brief pulse electroconvulsive therapy (ECT) to minimise its cognitive side-effects while retaining its efficacy. John O'Brien is Professor of Old Age Psychiatry in the Department of Psychiatry at the University of Cambridge. He is a National Institute for Health Research (NIHR) Emeritus Senior Investigator, Fellow of the Academy of Medical Sciences and the NIHR National Specialty Lead for Dementia. His research interests include the use of neuroimaging in dementia. Benjamin Underwood is a consultant old age psychiatrist at Cambridge and Peterborough NHS Foundation Trust. He is clinical director of the Windsor Research Unit in Cambridge, Dementia Clinical Lead for the NIHR Clinical Research Network (CRN) Eastern and NIHR CRN National Lead for Stratified Medicine in Dementia. Correspondence Dr Emad Sidhom. Email: es839@medschl.cam.ac.uk

First received 6 Feb 2020

Final revision 8 Apr 2020 Accepted 13 Apr 2020

\section{Copyright and usage}

(C) The Author(s), 2020. Published by Cambridge University Press on behalf of The Royal College of Psychiatrists. This is an Open Access article, distributed under the terms of the Creative Commons Attribution-NonCommercialShareAlike licence (http://creativecommons.org/licenses/by-nc-sa/4.0/), which permits non-commercial re-use, distribution, and reproduction in any medium, provided the same Creative Commons licence is included and the original work is properly cited. The written permission of Cambridge University Press must be obtained for commercial re-use. 
BOX 1 'Omic' technologies

The '-omic-' technologies increase substantially the number of proteins/genes that can be detected simultaneously to relate complex mixtures to complex effects in the form of gene/protein expression profiles.

Debnath 2010

Can stratified medicine be extended to other areas of medicine, and in particular to the greatest medical challenge of our times, dementia? There are already nearly a million people suffering from dementia in the UK and this number is set to double in the next 15 years (Matthews 2016). In this context any impact stratified medicine can achieve would be welcome. Relatively small effects of new treatments may have a significant impact: for example, it has been estimated that even relatively modest delays in the onset of Alzheimer's disease would decrease the numbers of those suffering from the disease by almost 40\% (Sloane 2002). In this context the attraction of using the latest scientific advances to enhance treatment is obvious.

\section{How has stratified medicine been applied in other medical specialties?}

The area of medicine perhaps most traditionally associated with stratified medicine is oncology. The targeting of breast cancers that are sensitive to oestrogen by tamoxifen, which selectively inhibits oestrogen receptors in tumour tissue, is one of the earliest and most successful examples of stratified medicine (Quirke 2017). Tamoxifen was first approved for use by the
US Food and Drug Administration in 1998. Further advances in the treatment of breast cancer build on this approach, for example the use of trastuzumab in tumours positive for human epidermal growth factor receptor (HER2) (Slamon 2001). In this way the prognosis of breast cancer has gradually improved to a point where the 5-year survival for women with breast cancer in the UK is now $90 \%$, a figure that has doubled in the past 40 years (Cancer Research UK 2020). Stratified medicine has made great advances in other areas of oncology and led to specific treatments. Examples include drugs used only in the context of specific mutations in lung cancer and melanoma (Flaherty 2012). The study of genetic influences on disease (genomic medicine) has led to further refinement of tumour classification in oncology, as well as producing a large patient data-set that can lead to new therapeutic targets (Soverini 2019).

Stratified medicine approaches in current clinical use are not restricted to cancer. Other examples include the use of antisense oligomers (which bind to specific genetic mutations) in a minority of patients with Duchenne muscular dystrophy and an increase in the number of drugs used for specific mutations of cystic fibrosis (Yu 2012).

In summary, in many areas of medicine, mutations can now be identified in specific genes that indicate which treatments are likely to be effective, meaning that stratified medicine approaches have immediate therapeutic benefit. There are also some examples of stratification of treatment from within psychiatric practice, with perhaps the most common being the titration of treatment for electroconvulsive therapy (ECT) against individual seizure threshold (Sackeim 1987; McCall 2000; National Institute for Health and Care Excellence 2003).

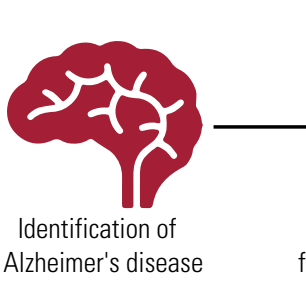
1906

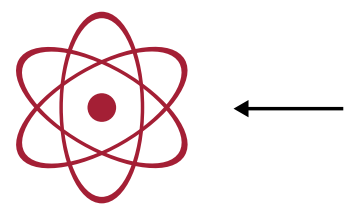

Pittsburgh compound B for amyloid PET 2004

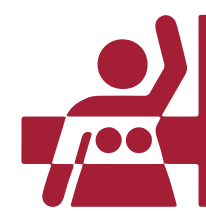

Tamoxifen identified for treatment of breast cancer 1967

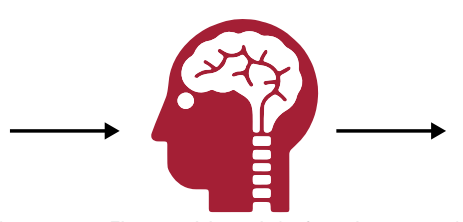

First positive trial of tacrine 1981

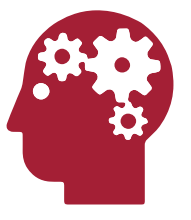

ApoE identified 1993

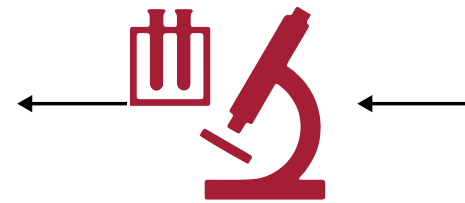

First study on CSF biomarkers 1995

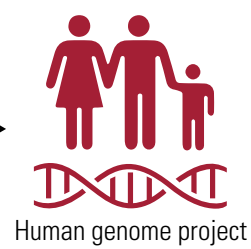

1990

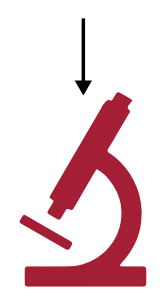

Discovery of APP 1991

Important milestones in stratified medicine and dementia.

APP, amyloid precursor protein; CSF, cerebrospinal fluid; PET, positron emission tomography. 


\section{What is the national context in the UK?}

The success of stratified medicine in oncology has understandably created interest in whether this approach might be successful in other fields. Efforts to foster the approach include a number of national initiatives, such as the Technology Strategy Board's Stratified Medicine Innovation Platform, launch of Cancer Research UK's Stratified Medicine Programme, creation of the UK Pharmacogenetics and Stratified Medicine Network and the Human Genome Project and National Phenome Centre. In 2014 the Association of the British Pharmaceutical Industry conducted a baseline evaluation of the state of stratified medicine outside of oncology in the UK using a review of available data, professional and industry interviews and a survey of drugs in development. This found that there was still marked variation in appreciation of stratified medicine. Most was still occurring in oncology, followed by infection, but in terms of pharmaceutical products in development which required an associated biomarker, those targeting the nervous system were the second biggest group, only beaten by antineoplastic drugs. The biggest barrier identified by respondents was the lack of companion diagnostic tests (Association of the British Pharmaceutical Industry 2014).

In the UK most clinical research is delivered within the National Health Service (NHS) via the National Institute for Health Research (NIHR) Clinical Research Network (CRN). The purpose of this organisation is to maximise the number of patients and clinicians taking part in research to deliver improved quality of care and to make the UK an effective and attractive place to perform clinical research. The organisation has proved remarkably effective. In 2018-2019, over 870000 people took part in CRN-supported studies in England (National Institute for Health Research 2020). The $\mathrm{CRN}$ has engaged in a project with the UK
Pharmacogenetics and Stratified Medicine Network to ensure that the $\mathrm{CRN}$ remains agile and ready to deliver on the challenges of stratified medicine. This has led to champions in stratified medicine in each disease area (including dementia) within the CRN, tracking of stratified medicine studies on the $\mathrm{CRN}$ portfolio and the establishment of the CRN Stratified Medicine Community. This has been further supplemented by special consideration of how stratified medicine might have an impact in specialties where it has not traditionally had a profile, including general practice and community healthcare.

\section{How might stratified medicine be applied to dementia?}

Stratified medicine is already applied in dementia care, including in clinical trials and current clinical practice (Table 1). Subtyping of dementia into diagnostic groups is important to target current symptomatic treatments. For example, cholinesterase inhibitors have a strong evidence base in Alzheimer's disease and Lewy body disease, but have not demonstrated benefit in frontotemporal dementia or Huntington's disease (Courtney 2004; Cubo 2006). This sort of clinical stratification is fairly crude, with substantial evidence of inaccurate diagnosis, possibly due in part to the need to rely on clinical diagnostic criteria with diagnostic tests of limited accuracy (Rizzo 2018). It is possible that the benefits of existing treatments could be further enhanced using stratified medicine. Donepezil (a symptomatic treatment for Alzheimer's and Lewy body disease) is metabolised in the liver by cytochromes CYP2D6 and CYP3A4, with the latter metabolising the drug to an inactive metabolite. Despite this, attempts to find differences in treatment in terms of outcome or side-effects by genetic variation in these cytochromes have not produced convincing results and so not entered clinical

Comparison of dementia and oncology in key areas of stratified medicine

\begin{tabular}{|c|c|c|}
\hline & Dementia & Oncology \\
\hline Clinical subtyping of diagnosis & Increasing use & High use \\
\hline Specificity of pre-mortem diagnosis & Low-medium & High \\
\hline Variable response to treatment depending on clinical group & Yes & Yes \\
\hline Variable response to treatment depending on biomarkers & $\begin{array}{l}\text { Varying response to treatment depending on diagnosis not } \\
\text { yet robustly tied to any biomarker }\end{array}$ & Yes \\
\hline Molecular, genetic and imaging stratification tools available & Yes, and increasing (Table 2) & Yes \\
\hline Genetic diagnosis & $\begin{array}{l}\text { Rare, though applicable to some forms of dementia, such } \\
\text { as Huntington's disease and familial Alzheimer's } \\
\text { disease }\end{array}$ & Increasingly common \\
\hline Specific histopathology & Yes (not usually available pre-mortem) & Yes (may be available pre-mortem \\
\hline Specific therapy targeted at individual-level variation & No & Yes \\
\hline Availability of disease-modifying treatment & No & Yes \\
\hline
\end{tabular}


practice (Lam 2009). The possibility of this sort of approach bearing fruit for existing treatments remains and it may also be applied to enhance any future treatments.

One obvious way of stratifying patients with a dementia syndrome is by diagnostic subtype, for example Alzheimer's disease, vascular dementia, Lewy body disease. Some of these diagnostic groups are unified by common pathology as well as clinical presentation: for example, Parkinson's disease dementia and Lewy body disease are both characterised by excess build up of the protein alpha-synuclein and so are known as synucleinopathies. However, other clinical diagnostic groups are not so coherent. For example, corticobasal degeneration and progressive supranuclear palsy are both caused by excess deposition of the protein tau. However, multisystem atrophy is a synucleinopathy, despite it being historically categorised, on clinical grounds, together with corticobasal degeneration and progressive supranuclear palsy (Erkkinen 2018). Understanding this distinction is essential for the appropriate use in clinical trials of any drug targeted at the underlying pathological protein (Jabbari 2019). Similarly, in frontotemporal dementia there are a variety of potential underlying genetic abnormalities, including in genes for tau and progranulin and in the CHMP2B, VCP, TDP-43, FUS and C9orf72 genes, and there may be others that are as yet unknown (Ferrari 2019). As our understanding of disease advances, traditional classification systems are likely to need significant revision, which will be a challenge to clinicians, who will need frequently to adapt clinical practice in the light of advances in scientific understanding.

Dilemmas of diagnostic classification are relevant not only to rare causes of dementia or determinate genes inherited in a dominant or recessive fashion. The risk variants of the $A P O E$ gene are well established in Alzheimer's disease and now more than 20 additional genetic loci that increase the risk of Alzheimer's disease have been robustly identified through genome-wide association studies. These have been subsequently analysed to identify potential common metabolic pathways, which include lipid metabolism, endocytosis and the inflammatory response (Freudenberg-Hua 2018; Nikolac Perkovic 2019). Even in Lewy body disease, where the genetic basis has historically been less well understood, interest is growing in a variant of the enzyme glucocerebrosidase as a genetic risk factor with a view to refining treatment (Blandini 2019). What bearing any genetic variant has on a particular treatment outcome is not clear, but it could influence the outcome of any clinical trial. Genetic testing is still rarely performed in memory clinics and histopathology is not usually available until postmortem.

\section{What might be the specific problems in applying stratified medicine to dementia?}

Experience from other disciplines is instructive in relation to the sort of problems that might challenge adoption of stratified medicine in dementia care. In oncology, barriers have included uptake of molecular testing and subsequent clinical uptake (Ersek 2018). In the context of Alzheimer's disease, if a treatment became available for which efficacy was shown only in those with a particular PET scan or CSF biomarkers for example, how many UK older people's mental health services would be currently able to offer PET or lumbar puncture as part of routine diagnostic workup? What impact would this have on waiting times? It would create potentially significant extra clinical demand. For example, in the county of Cambridgeshire we have over 8000 people with dementia and nearly 3000 new dementia assessments each year. The need for biomarkers could generate a very large new demand on scanning time, for example, which would need organising and funding. Even 1000 new PET scans at a notional cost of $£ 1000$ each would result in a $£ 1$ million increase in costs. It is possible that significant increase in cost may be easier to consider in potentially curative treatment for young patients, but less so for drugs that may only be disease-modifying or symptomatic treatments in elderly people, and this raises the possibility of age discrimination. How would one deal with patients who were unable to undergo the diagnostic procedure? Would treatment be restricted unless accompanied by a positive test? This might be a bigger problem in older and more frail patients, whose ability to tolerate invasive testing may be diminished.

Although in some ways these problems would be welcome as they would signify progress and might also stimulate more accurate diagnostic assessment, they are still worthy of consideration at an early stage of development of any drug tied to a biomarker.

Table 1 compares dementia and oncology in a number of key areas relevant to stratified medicine: differences persist but there are significant areas of overlap.

\section{What other tests may be clinically available to stratify disease populations in dementia?}

Increasingly amyloid (and potentially tau) PET imaging and analysis of the amyloid/tau ratio in CSF have been used to try to identify people with Alzheimer's disease for enrolment into clinical 
TABLE 2 Available potential imaging, molecular and genetic techniques for stratification in dementia clinical trials

\begin{tabular}{|c|c|c|}
\hline Stratification tool & Type & Use \\
\hline Autosomal dominant gene mutations & Genetic deterministic variant & $\begin{array}{l}\text { Identifying those with familial causes of dementia (Huntington's disease } \\
\text { Alzheimer's disease, frontotemporal dementia) }\end{array}$ \\
\hline Testing for other risk variants (e.g. ApoE) & Genetic risk variant & Risk stratification for Alzheimer's disease. \\
\hline${ }^{123}$-ioflupane radioactive tracer $\left(\mathrm{DaTSCAN}{ }^{\circledR}\right.$ ) & Imaging & $\begin{array}{l}\text { Specific diagnosis of Lewy body disease and Parkinson's disease } \\
\text { dementia }\end{array}$ \\
\hline MIBG myocardial scintigraphy & Imaging (cardiac nuclear medicine) & $\begin{array}{l}\text { Specific diagnosis of Lewy body disease and Parkinson's disease } \\
\text { dementia }\end{array}$ \\
\hline Amyloid and tau PET & Imaging of cerebral amyloid or tau burden & Identifying amyloid or tau pathology consistent with underlying diagnosis \\
\hline Structural CT or MRI & Imaging & $\begin{array}{l}\text { Subtyping dementia (vascular, Alzheimer's disease, Lewy body disease, } \\
\text { etc.) }\end{array}$ \\
\hline FDG PET & Imaging & Subtyping of dementia \\
\hline Tau/amyloid CSF ratio & CSF biomarker & Specific diagnosis of sporadic Alzheimer's disease \\
\hline Detailed neuropsychiatric assessment & Neuropsychiatric assessment & Subtyping of dementia \\
\hline Metabonomics & 'Omic' technology & Diagnosis and staging of dementia \\
\hline
\end{tabular}

ApoE, apolipoprotein E; CSF, cerebrospinal fluid; CT, computed tomography; FDG, fluorodeoxyglucose; MIBG, ${ }^{123}$-meta-iodobenzylguanidine; MRI, magnetic resonance imaging; PET, positron emission tomography.

trials (Klunk 2004), and new fluid biomarkers such as neurofilament light protein and neurogranin are appearing (Molinuevo 2018). Such finding may be changing trial design. In 2019, one-third of the phase II and phase III drug trials in Alzheimer's disease in the USA were using either amyloid PET of CSF biomarkers (Cummings 2019). It may be the case that trials of therapies targeted at amyloid in an unstratified group would have been liable to type 2 error due to the trial being underpowered to detect any effect. This is one possible explanation for why so many studies in Alzheimer's disease have failed and raises the possibility that already discarded treatments may have had utility in better-defined (for example amyloid-positive) populations. Increasing use of stratified medicine may improve this, particularly in investigation of drugs that target beta-amyloid. Some of these cross the blood-brain barrier and decrease cerebral amyloid burden, but despite stratifying to only those patients where cerebral amyloid can be demonstrated, the trials have been largely negative, although some signal of benefit may exist (Mo 2017). Even the relative failure of amyloid-targeting therapies has been helped by stratification - failure is not likely to be due to the patients not having Alzheimer's disease or an amyloid burden and we can therefore be more certain that the results are truly negative rather than a type 2 error arising from using these treatments in a more heterogeneous group of patients. Such advances hold out the prospect of combining these approaches, not least to predict progression and aid diagnosis. For example, one can envisage the possibility of combining genetic profiling with imaging and CSF biomarkers to identify individuals with dementia at an early stage and target treatment accordingly. Table 2 lists currently available imaging, molecular and genetic techniques that could be used to stratify dementia populations.

Where drugs are targeting a specific molecular pathway then stratification is likely to enhance efficacy and avoid exposing patients to side-effects with no expectation of benefit. However, this is not true of all potential therapeutic approaches in dementia. For example, work in the department of clinical neurosciences at the University of Cambridge, UK, has pioneered novel therapeutic approaches targeting protein turn-over. One of these is to prevent activation of the unfolded protein response, maintain synthesis of proteins essential for neuronal health and ameliorate disease (Mercado 2017). Another is to up-regulate the cellular process of autophagy to enhance the degradation of pathogenic protein species (Ravikumar 2004). These approaches are made more attractive by the fact that 'repurposed' drugs already in clinical use, for example trazodone, can target these pathways in laboratory models. Although it is possible that stratification of patients may yet play a role, these approaches target pathological pathways that may be common to many neurodegenerative diseases and therefore stratification by, for example, precise diagnosis may be less important.

Stratified medicine has been most successful in oncology, where specific genetic mutations or other characteristics of the tumour provide a specific therapeutic target. This may be less relevant in dementia, where less than $1 \%$ of cases have a specific genetic underpinning. Even where it does occur, for example in Huntington's disease, there may be challenges in specifically targeting mutant rather than wild-type protein - and this may be an important safety consideration. New approaches to this disease, such as the use of intrathecal oligonucleotides and other ways of suppressing mutant 
MCO answers

$1 \mathrm{c} \quad 2 \mathrm{a} \quad 3 \mathrm{~d} \quad 4 \mathrm{~b} \quad 5 \mathrm{e}$ huntingtin production, are now appearing in clinical trials (Kaemmerer 2019; Tabrizi 2019). Although stratified medicine is exciting at this level of specificity, more fundamental methods of stratification at the other end of the spectrum of specificity should not be ignored. For example, there has long been an interest in sexual dimorphism in the context of Alzheimer's disease and, although very broad, this still may be an important consideration in designing optimal treatment (Hampel 2018).

The last area in which stratified medicine may make an impact is in the economic modelling of trial design. Before commencing trials various trial designs (including 'all-comers' approaches or the application of stratified medicine) can be compared in terms of cost and probability of regulatory success to create an expected net present value - a combination of the present value and the probability of a trial being successful. This approach has been applied to Alzheimer's disease, and in particular the use of APOE status in some anti-amyloid studies, where efficacy is less likely and side-effect burden is higher in those with an APOE4 genotype. This analysis demonstrated a sixfold increase in expected net present value for stratified over allcomers designs (Trusheim 2011).

\section{Conclusions}

Stratified medicine is an approach that seeks to personalise treatment on the basis of increasing genetic and pathological knowledge. It has already brought benefit in several areas of medicine and there is enthusiasm to translate these approaches to more disease areas, including dementia. Stratified medicine is applicable to dementia because of the increasing recognition of genetic causes and contribution, and the ability to measure protein markers of pathology such as amyloid and tau using brain imaging and through CSF analysis. The increasing use of stratified medicine in dementia may have an impact on clinical practice not only in terms of trial recruitment and potential new treatments but also in refining things as fundamental as diagnostic classification. Although there are a number of challenges to applying stratified medicine to dementia, there are both narrow and broader opportunities in doing so. The support this is receiving in the UK is welcome.

\section{Authors contributions}

All authors contributed to the main idea, the writing and the final manuscript.

\section{Declaration of interest}

J.O'B. reports personal fees from TauRx, personal fees from Axon, personal fees from GE Healthcare, grants and personal fees from Avid/Lilly, personal fees from Eisai, grants from Alliance Medical, personal fees from Roche and grants from Merck, outside the submitted work.

ICMJE forms are in the supplementary material, available online at https://doi.org/10.1192/bja.2020. 28.

\section{References}

National Institute for Health and Care Excellence (2003) Guidance on the Use of Electroconvulsive Therapy (Technology Appraisal Guidance TA59). NICE.

Association of the British Pharmaceutical Industry (2014) Stratified medicine in the NHS. ABPI (https://www.abpi.org.uk/publications/stratifiedmedicine-in-the-nhs/). Accessed 29 Jan 2020.

Academy of Medical Sciences (2020) Stratified medicine. Academy of Medical Sciences (https://acmedsci.ac.uk/policy/policy-projects/ Stratified-medicine). Accessed 29 Jan 2020.

Cancer Research UK (2020) Breast cancer statistics. Cancer Research UK (https://www.cancerresearchuk.org/health-professional/cancer-statistics/statistics-by-cancer-type/breast-cancer\#heading-Three). Accessed 30 Jan 2020

National Institute for Health Research (2020) Clinical Research Network. NIHR (https://www.nihr.ac.uk/explore-nihr/support/clinical-research-network.htm). Accessed 30 Jan 2020

Berrios GE (1990) Alzheimer's disease: a conceptual history. International Journal of Geriatric Psychiatry, 5: 355-65.

Blandini F, Cilia R, Cerri S, et al (2019) Glucocerebrosidase mutations and synucleinopathies: toward a model of precision medicine. Movement Disorders, 34: 9-21.

Blennow K, Zetterberg H (2015) The past and the future of Alzheimer's disease CSF biomarkers - a journey toward validated biochemical tests covering the whole spectrum of molecular events. Frontiers in Neuroscience, 9: 345 .

Corder EH, Saunders AM, Strittmatter WJ, et al (1993) Gene dose of apolipoprotein E type 4 allele and the risk of Alzheimer's disease in late onset families. Science, 261: 921-3.

Courtney C, Farrell D, Gray R, et al (2004) Long-term donepezil treatment in 565 patients with Alzheimer's disease (AD2000): randomised doubleblind trial. Lancet, 363: 2105-15.

Cubo E, Shannon KM, Tracy D, et al (2006) Effect of donepezil on motor and cognitive function in Huntington disease. Neurology, 67: 1268-71.

Cummings J, Lee G, Ritter A, et al (2019) Alzheimer's disease drug development pipeline: 2019. Alzheimer's and Dementia, 5: 272-93.

Debnath M (2010) Omics Technology. In Molecular Diagnostics: Promises and Possibilities (eds M Debnath M, GB Prasad, PS Bisen): 11-31. Springer.

Erkkinen MG, Kim MO, Geschwind MD (2018) Clinical neurology and epidemiology of the major neurodegenerative diseases. Cold Spring Harbor Perspectives in Biology, 10(4): a033118.

Ersek JL, Black LJ, Thompson MA, et al (2018) Implementing precision medicine programs and clinical trials in the community-based oncology practice: barriers and best practices. American Society of Clinical Oncology Educational Book, 38: 188-96.

Ferrari R, Manzoni C, Hardy J (2019) Genetics and molecular mechanisms of frontotemporal lobar degeneration: an update and future avenues. Neurobiology of Aging, 78: 98-110.

Flaherty KT, Robert C, Hersey P, et al (2012) Improved survival with MEK inhibition in BRAF-mutated melanoma. New England Journal of Medicine, 367: 107-14

Freudenberg-Hua Y, Li W, Davies P (2018) The role of genetics in advancing precision medicine for Alzheimer's Disease-A narrative review. Frontiers in Medicine, 5: 108. 
Goate A, Chartier-Harlin MC, Mullan M, et al (1991) Segregation of a missense mutation in the amyloid precursor protein gene with familial Alzheimer's disease. Nature, 349: 704-6.

Hampel H, Vergallo A, Giorgi FS, et al (2018) Precision medicine and drug development in Alzheimer's disease: the importance of sexual dimorphism and patient stratification. Frontiers in Neuroendocrinology, 50: $31-51$.

Jabbari E, Holland N, Chelban V, et al (2019) Diagnosis across the spectrum of progressive supranuclear palsy and corticobasal syndrome. JAMA Neurology. Available from: https://doi.org/10.1001/ jamaneurol.2019.4347.

Jordan VC (2006) Tamoxifen (ICI46,474) as a targeted therapy to treat and prevent breast cancer. British Journal of Pharmacology [Epub ahead of print], Dec 20. Available from: https://doi.org/10.1038/sj.bjp.0706399.

Kaemmerer WF, Grondin R (2019) The effects of huntingtin-lowering: what do we know so far? Degenerative Neurological and Neuromuscular Disease, 9: 3-17.

Klunk WE, Engler H, Nordberg A, et al (2004) Imaging brain amyloid in Alzheimer's disease with Pittsburgh compound-B. Annals of Neurology, 55: 306-19.

Lam B, Hollingdrake E, Kennedy JL, et al (2009) Cholinesterase inhibitors in Alzheimer's disease and Lewy body spectrum disorders: the emerging pharmacogenetic story. Human Genomics, 4: 91-106.

Lenz C, Steffen JA, Engelsberg A (2013) Molecular testing guidelines for selection of lung cancer patients for ALK tyrosine kinase inhibitors. Journal fur Pharmakologie und Therapie, 22: 45-8.

Matthews FE, Stephan BC, Robinson L, et al (2016) A two decade dementia incidence comparison from the Cognitive Function and Ageing Studies I and II. Nature Communications, 7: 11398.

McCall WV, Reboussin DM, Weiner RD, et al (2000) Titrated moderately suprathreshold vs fixed high-dose right unilateral electroconvulsive therapy. Archives of General Psychiatry, 57: 438-44.

Mercado G, Hetz C (2017) Drug repurposing to target proteostasis and prevent neurodegeneration: accelerating translational efforts. Brain, 140: $1544-7$.

Michels S, Wolf J (2016) Stratified treatment in lung cancer. Oncology Research and Treatment, 39: 760-6.

Mo JJ, Li JY, Yang Z, et al (2017) Efficacy and safety of anti-amyloid- $\beta$ immunotherapy for Alzheimer's disease: a systematic review and network meta-analysis. Annals of Clinical and Translational Neurology, 4: $931-42$.

Molinuevo JL, et al (2018) Current state of Alzheimer's fluid biomarkers. Acta Neuropathologica, 136: 821-53.

Moraes F, Góes A (2016) A decade of human genome project conclusion: scientific diffusion about our genome knowledge. Biochemistry and Molecular Biology Education, 44: 215-23.
Motter R, Vigo-Pelfrey C, Kholodenko D, et al (1995) Reduction of $\beta$-amyloid peptide42 in the cerebrospinal fluid of patients with Alzheimer's disease. Annals of Neurology, 38: 643-8.

Nikolac Perkovic M, Pivac N (2019) Genetic markers of Alzheimer's disease. Advances in Experimental Medicine and Biology, 1192: 27-52.

Quirke VM (2017) Tamoxifen from failed contraceptive pill to best-selling breast cancer medicine: a case-study in pharmaceutical innovation. Frontiers in Pharmacology, 8: 620.

Ravikumar B, Vacher C, Berger Z, et al (2004) Inhibition of mTOR induces autophagy and reduces toxicity of polyglutamine expansions in fly and mouse models of Huntington disease. Nature Genetics, 36: 585-95.

Rizzo G, Arcuti S, Copetti M, et al (2018) Accuracy of clinical diagnosis of dementia with Lewy bodies: a systematic review and meta-analysis. Journal of Neurology, Neurosurgery and Psychiatry, 89: 358-66.

Sackeim HA, Decina P, Kanzler M, et al (1987) Effects of electrode placement on the efficacy of titrated, low-dose ECT. American Journal of Psychiatry, 144: 1449-55.

Slamon DJ, Leyland-Jones B, Shak S, et al (2001) Use of chemotherapy plus a monoclonal antibody against HER2 for metastatic breast cancer that overexpresses HER2. New England Journal of Medicine, 344: 783-92.

Sloane PD, Zimmerman S, Suchindran C, et al (2002) The public health impact of Alzheimer's disease, 2000-2050: potential implication of treatment advances. Annual Review of Public Health, 23: 213-31.

Soverini S, Abruzzese E, Bocchia M, et al (2019) Next-generation sequencing for BCR-ABL1 kinase domain mutation testing in patients with chronic myeloid leukemia: a position paper. Journal of hematology \& oncology, 12: 131

Stelzmann RA, Schnitzlein HN, Murtagh FR (1995) An English translation of Alzheimer's 1907 paper, "Über eine eigenartige Erkankung der Hirnrinde". Clinical Anatomy, 8: 429-31

Strittmatter WJ, Saunders AM, Schmechel D, et al (1993) Apolipoprotein E: high-avidity binding to beta-amyloid and increased frequency of type 4 allele in late-onset familial Alzheimer disease. PNAS, 90: 1977-81.

Summers WK, Viesselman JO, Marsh GM, et al (1981) Use of THA in treatment of Alzheimer-like dementia: pilot study in twelve patients. Biological Psychiatry, 16: 145-53.

Tabrizi SJ, Leavitt BR, Landwehrmeyer GB, et al (2019) Targeting huntingtin expression in patients with Huntington's disease. New England Journal of Medicine, 380: 2307-16.

Trusheim MR, Burgess B, Hu SX, et al (2011) Quantifying factors for the success of stratified medicine. Nature Reviews Drug Discovery, 10: 817-33.

Venter JC, Adams MD, Myers EW, et al (2001) The sequence of the human genome. Science, 291: 1304-51.

Yu H, Burton B, Huang CJ, et al (2012) Ivacaftor potentiation of multiple CFTR channels with gating mutations. Journal of Cystic Fibrosis, 11(3): 237-45. 


\section{MCQs}

Select the single best option for each question stem

1 Stratified medicine refers to:

a treatment with more than one approach

b dose stratification and titration based on response

c classification of patients according to subclass of disease or the likelihood of responding to a particular therapy

d creation of medicines or medical devices that have broad application across populations and diseases

e current guidelines for treatment.
2 The clinical stratification of neurodegenerative disorders is:

a crude, owing to lack of precise diagnostic tests

b precise according to recent classifications

c dependent on the response to acetylcholinesterase inhibitors or NMDA receptor antagonists

d irrelevant to current antidementia medication

e often precisely mapped onto pathology and underlying genetics.

3 The Department of Clinical Neurosciences at the University of Cambridge, UK, has pioneered:

a novel acetylcholine esterase inhibitors

b newer NMDA receptor antagonists

c megadoses of combinations of acetylcholinesterase inhibitors and NMDA receptor antagonists

d preventing the activation of the unfolded protein response

e enhancing the activation of the unfolded protein response.
4 An example of current personalised treatment in psychiatric practice is:

a the use of acetylcholinesterase inhibitor dose titration

$b$ the use of ECT dose titration

c the use of NMDA receptor antagonist dose titration

$d$ the use of treatment algorithms for antipsychotics

e not yet available.

5 The application of stratified medicine maybe less important in:

a CSF biomarkers

b drugs targeting beta-amyloid

c drugs targeting tau bodies

d ApoE subtypes

e drugs targeting the unfolded protein response or upregulating autophagy. 